\title{
Monitor unit calculation for radiotherapy treatments using the Monte Carlo method
}

\author{
Hidmer Laulate* \\ Programa de Engenharia Nuclear, COPPE/UFRJ \\ Rio de Janeiro, Brazil \\ E-mail: hmelgarejo@nuclear.ufrj.br
}

Artur Ferreira de Menezes

Programa de Engenharia Nuclear, COPPE/UFRJ

Rio de Janeiro, Brazil

Juraci Passos dos Reis Junior

Programa de Engenharia Nuclear, COPPE/UFRJ

Rio de Janeiro, Brazil

\section{Leonardo Peres da Silva}

Programa de Engenharia Nuclear, COPPE/UFRJ

Rio de Janeiro, Brazil

\section{Ademir Xavier da Silva}

Programa de Engenharia Nuclear, COPPE/UFRJ

Rio de Janeiro, Brazil

\begin{abstract}
This study aims to develop a methodology to quantify and assess the monitor unit (MU) for a radiotherapy treatment simulation using the Monte Carlo method. For this study was reproduced a standard treatments considering that the patient has low risk prostate cancer. In low risk prostate cancer, the Clinical Target Volume (CTV) should be restricted to the prostate only and the Planning Target Volume (PTV) was considered $1 \mathrm{~cm}$ beyond the CTV. An additional $0.6 \mathrm{~cm}$ margin is added to account for penumbra. In this treatment we use $6 \mathrm{MeV}$ photons, the four-field box technique and 2 Gy daily fractions, $50 \mathrm{cGy}$ for each treatment field. The irradiations were performed using the Siemens Oncor Expression linear accelerator belonging to the service of the Clinicas Oncologicas Integradas (COI/RJ), modeled and validated using the MCNPX code.
\end{abstract}

X Latin American Symposium on Nuclear Physics and Applications (X LASNPA),

1-6 December 2013

Montevideo, Uruguay

\footnotetext{
*Speaker.
} 


\section{Introduction}

In conformal radiotherapy is used a software called the Treatment Planning System (TPS) to perform treatment planning, in order to obtain conformal field to the treatment volume, that is shaped to match the target volume, allowing to increase the radiation dose delivered to the tumor, to improve treatment outcomes and reduce the dose of radiation that reaches the normal tissues [1]. Monitor Unit calculation in Treatment Planning Systems is done using dose calculations algorithms for the release of the prescribed dose to the patient [2]. ICRU Report 62 [3] as the work of Mijnheer [4] and Wambersie [5] proposed that the uncertainty in the delivered dose must not be greather than $\pm 3.5 \%$. These uncertainties can be introduced during treatment (including the calibration of the treatment unit) or during the process of determination of monitor units. Errors in the determination of the MU, arising from the planning phase, could potentially affect the entire course of treatment. The limits on deviations in the calculation of monitor unit in clinical practice is $3.5 \%$ [6]. A procedure for calculating the Monitor Unit is necessary to ensure that the delivered is accurate. In this work, we used a methodology for monitor unit calculation using the MCNPX code, for a prostate cancer treatment based in the MAX06 phantom, to ensure that the prescribed dose used in treatment of prostate cancer is delivered properly. Using the four field technique, cerrobend blocks are used for shaping the radiation beam, the prescribed dose is $50 \mathrm{cGy}$ at isocenter for each treatment field.

\section{Methods and Materials}

\subsection{Monte Carlo Modeling of the Siemens Oncor Expression Linear Accelerator}

The main components of Siemens ONCOR Expression linear accelerator belonging to the radiotherapy service of the Clínicas Oncológicas Integradas (COI), have been modeled using the Monte Carlo code MCNPX [7], from the combination of different surfaces such as: parallelepipeds, cylinders, pyramids and plans. The accelerator was validated applying the phase space technique, based on the dosimetric parameters: Percentage depth dose (PDD), the beam profiles and the photon spectrum, obtained for a square field of dimensions $10 \times 10 \mathrm{~cm}^{2}$ in a water tank of dimensions $30 \times 30 \times 30 \mathrm{~cm}^{3}$, considering the source surface distance (SSD) of $100 \mathrm{~cm}$ and the photon energy equal to $6 \mathrm{MeV}$. The depth of maximum dose $\left(d_{\max }\right)$ is $1.5 \mathrm{~cm}$. The generation of the phase space was performed in Orion Cluster System, belonging to the Neutron Laboratory of the Institute of Radiation Protection and Dosimetry (IRD).

\subsection{The MAX06 Phantom}

The MAX (Male Adult voXel) phantom was developed by Kramer [8], based on segmented images of an adult male patient VOX_TISS8 provided by Zubal in 2001 [9], has its masses of organs and tissues in agreement with the reference values of ICRP 89 [10]. The MAX06 phantom [11] is an update of the MAX phantom to improve compatibility with the ICRP 103 [12]. Since it is included structures such as bronchi, the lymph nodes and the prostate that were not available in the original phantom. The skeleton of the new phantom was subdivided into compact, spongy, yellow bone marrow and cartilage, adding even more to the skeletal structure heterogeneities. There were also changes in the voxel edge of $3.6 \mathrm{~mm}$ to $1.2 \mathrm{~mm}$ for the MAX06 phantom, this reduction 
causes the voxels increase in the order of 27 times. Figure 2.8 shows a cross section of the MAX06 phantom.

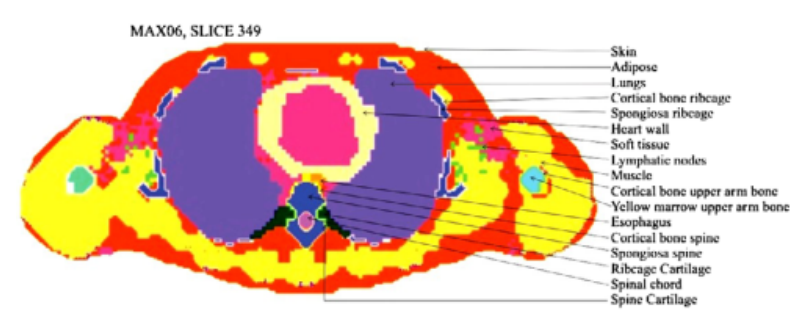

Figure 1: The MAX06 phantom

\subsection{Conformal Radiotherapy for Prostate Cancer}

This study was simulated a treatment of prostate cancer at low risk so that both the gross tumor volume (GTV) as the clinical target volume (CTV) are restricted to prostate only. In general, in this type of procedure is added a $1 \mathrm{~cm}$ margin around the CTV to define the PTV, to compensate possible variations both in the daily patient positioning as anatomical changes during treatment. To consider the penumbra due to the collimator block [13] is necessary to add a margin of $0.6 \mathrm{~cm}$.

For irradiation was used four field technique considering: one anterior field $\left(0^{\circ}\right)$, another posterior

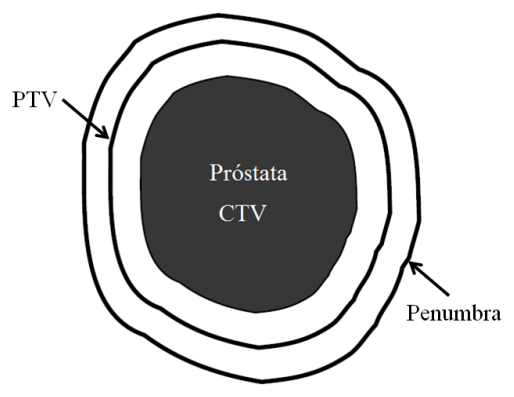

Figure 2: CTV-PTV margin: $1 \mathrm{~cm}$, penumbra: $0.6 \mathrm{~cm}$

$\left(180^{\circ}\right)$ and two lateral fields $\left(90^{\circ}\right.$ and $\left.270^{\circ}\right)$, equally weighted. The radiation beam is shaped using blocks cerrobend.
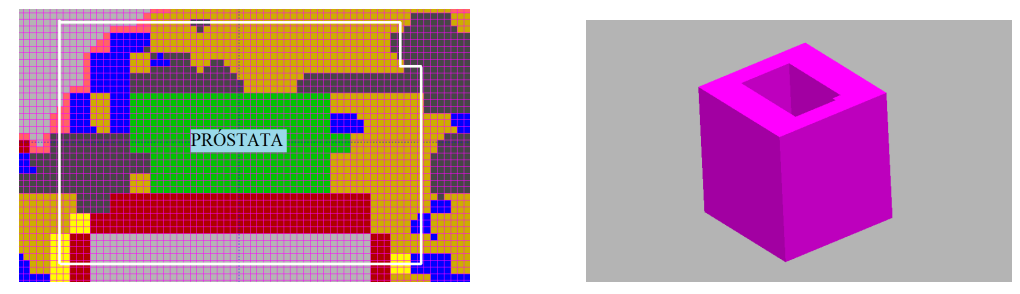

Figure 3: Margin around the prostate to model the collimator block in the plane of the isocenter

Treatment was administered at a daily dose of $2 \mathrm{~Gy}$, the sum of $50 \mathrm{cGy}$ for each treatment field used. To ensure proper delivery of the prescription dose in the isocenter of treatment was necessary 
to perform the calibration of the monitor unit (MU), determining the number of photons arriving at the position of the isocenter treatment to ensure that the desired dose is delivered.

\subsection{Monitor Unit Calculation}

For the calculation of the monitor unit for the treatment of prostate cancer using the four-field technique presented in this work, the methodology presented by Reis Junior [14]. The calculation for the monitor unit (MU) was performed for a source-surface distance (SSD) of $100 \mathrm{~cm}$, a water phantom of 30x30x30 $\mathrm{cm}^{3}$, a collimated field defined by cerrobend block, for each angle of treatment. A technique for generating phase space provided by MCNPX code was used to simulate the source dimensions $10 \times 10 \mathrm{~cm}^{2}$ located above the collimator blocks.

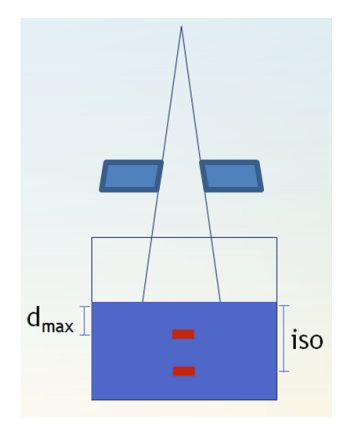

Figure 4: Scheme used to calculate the monitor unit

To optimize the computational time the doses were determined by tally $F 6(\mathrm{MeV} / \mathrm{g})$ of MCNPX code, using as detector cells two voxel of dimensions $0.15 \times 0.15 \times 0.15 \mathrm{~cm}^{3}$ equal to the MAX06 voxel phantom modified, and positioned at the depth of maximum dose $\left(d_{\max }=1.5 \mathrm{~cm}\right)$ and the depth of isocenter (iso), located in the center of mass of the prostate.

\begin{tabular}{c|c}
\hline \hline Treatment Angle & Isocenter $(\mathrm{cm})$ \\
\hline $0^{\circ}$ & 9.89 \\
$90^{\circ}$ & 20.17 \\
$180^{\circ}$ & 16.34 \\
$270^{\circ}$ & 22.13 \\
\hline \hline
\end{tabular}

Table 1: Depth of isocenter for each treatment angle.

For each treatment field, doses were determined using the $F 6_{\text {dmax }}(\mathrm{MeV} / \mathrm{g})$ and $F 6_{\text {iso }}(\mathrm{MeV} / \mathrm{g})$ commands to the above depths (Table 1). Doses were subsequently converted to gray (Gy) using the equations 2.1 and 2.2:

$$
\begin{gathered}
D_{1 \text { photon }}\left(d_{\max }\right)=F 6_{d \max }\left(\frac{M e V}{g}\right)=F 6_{d \max } \frac{1.602 \times 10^{-10} \mathrm{~J}}{10^{-3} \mathrm{Kg}}=F 6_{d \max } x 1.602 \times 10^{-10} \mathrm{~Gy} \\
D_{1 \text { photon }}(\text { iso })=F 6_{\text {iso }}\left(\frac{\mathrm{MeV}}{\mathrm{g}}\right)=F 6_{\text {iso }} \frac{1.602 \times 10^{-10} \mathrm{~J}}{10^{-3} \mathrm{Kg}}=F 6_{\text {iso }} x 1.602 \times 10^{-10} \mathrm{~Gy}
\end{gathered}
$$


At first the dose $\left(D_{P}\right)$ was prescribed at a depth of maximum dose $\left(d_{\max }\right)$ and the number of photons required to deliver $D_{P}$ was calculated by equation 2.3 .

$$
\text { Numberof photons }=\frac{D_{P}}{D_{1 \text { photon }}\left(d_{\max }\right)}
$$

For the treatment was prescribed a dose equal to $50 \mathrm{cGy}$ at isocenter for each field considered. Once the linear accelerator used was calibrated at $1 \mathrm{MU} \equiv 1 \mathrm{cGy}$ under reference conditions mentioned above, the prescription of $50 \mathrm{cGy}$ at the depth of maximum dose is $50 \mathrm{MU}$, which leads to the conclusion that the unit monitor, $M U_{d \max }$, at the depth of maximum dose, $d_{\max }$, is numerically equal to the prescribed dose, $D_{P}$. Naturally, the dose at the isocenter $\left(D_{i s o}\right)$ will be given by equation 2.4, where the number of photons is related to the amount of photons required to deliver the prescribed dose $\left(D_{P}\right)$ at the depth of maximum dose, $d_{\max }$.

$$
D_{\text {iso }}=D_{1 \text { photon }}(\text { iso }) x \text { Numberof photons }
$$

Therefore, to ensure that the dose to the isocenter of the injury is numerically equal to the initially prescribed dose at the depth of maximum dose ( $50 \mathrm{cGy})$, it will be necessary to increase the number of monitor units, by the fact that fewer photons arrive at greater depths due to attenuation in the material (water). The Monitor Unit $\left(M U_{\text {iso }}\right)$ required to administration of the prescribed dose at the depth of isocenter is numerically equal to the product of the prescribed dose $D_{P}\left(M U_{d \max }\right)$, and the ratio between the prescribed dose at the depth of maximum dose $\left(D_{P}\right)$ and the dose at the isocenter $\left(D_{\text {iso }}\right)$, as described in the equation 2.5.

$$
M U_{i s o}=M U_{d \max } x \frac{D_{P}}{D_{\text {iso }}}
$$

\section{Results}

In Table 2 are shown the monitor unit (MU) calculated using the methodology of section 2.4 as well as that obtained from the Prowess Panther treatment planning system (TPS) belonging to the $\mathrm{COI}$, to ensure that the prescribed dose is properly delivered in the depth of isocenter. The results shown that the monitor units obtained using the TPS and Monte Carlo simulation increase as the depth of the isocenter.

\begin{tabular}{c|c|c|c|c}
\hline \hline Treatment Angle & Isocenter $(\mathrm{cm})$ & MU calculated & MU TPS & Percentage Relative Error \\
\hline $0^{\circ}$ & 9.89 & 78.75 & 81.1 & 2.89 \\
$90^{\circ}$ & 20.17 & 145.51 & 149.2 & 2.47 \\
$180^{\circ}$ & 16.34 & 115.50 & 119.2 & 3.10 \\
$270^{\circ}$ & 22.13 & 164.01 & 167.2 & 1.90 \\
\hline \hline
\end{tabular}

Table 2: Monitor Unit for the simulated treatment.

The results generated using the Monte Carlo simulations remained slightly below the respective values obtained using the Prowess Panther treatment planning system. As can be seen in Table 
2 the maximum difference obtained by comparing the values generated using the MCNPX code and the treatment planning system was $3.1 \%$ for the incidence angle equal to $180^{\circ}$. The results for the other angles showed smaller relative differences.

\section{Conclusions}

After analyzing the results, it can be seen that the relative differences obtained by comparing the values of monitor units generated using the treatment planning system and the Monte Carlo method, remained at levels below $3.1 \%$, which is within the uncertainties accepted in clinical practice [6]. The results obtained from the comparative study between the values of monitor units, considering the linear accelerator modeled using the Monte Carlo method and the Planning System Prowess Panther proved satisfactory within the limits of tolerance in dose delivery using the technique of radiotherapy. This agreement validates the methodology for the calculation of monitor units using the Monte Carlo method presented in this study.

\section{References}

[1] S. R. Mahdavi, H. Rezaeejam, A.S. Hosntalab, A. Mostaar, M. Motamedi, Conformal fields in prostate radiotherapy: A comparison between measurement, calculation and simulation, Journal of Cancer Research and Therapeutics 8, 34 (2012).

[2] J. Chan, D. Russell, V. G. Peters, T. J. Farrell, Comparison of monitor unit calculations performed with a $3 D$ computerized planning system and independent hand calculations: Results of three years clinical experience, Journal of Applied Clinical Medical Physics 3, 293 (2002).

[3] ICRU Report 62, Prescribing, recording and reporting photon beam therapy, Washington, 1999.

[4] B. J. Mijnheer, J. J. Battermann, A. Wambersie, What degree of accuracy is required and can be achieved in photon and neutron therapy?, Radiother Oncol 8, 237 (1987).

[5] A. Wambersie, J. Van Dam, G. Hank, B. J. Mijnheer, J. J. Battermann, What accuracy is needed in dosimetry?, Tecdoc 734: IAEA, Vienna, 11(1994).

[6] M. R. Muller, L. N Rodrigues, M. A. Silva, development and implementation of a software for assessment of calculations in radiotherapy monitors units Radiol Bras 38, 415 (2005).

[7] J. P. Reis Junior, H. Salmon, A. F. Menezes, G. A. Pavan, L.A.R. Rosa, Simulation of Siemens ONCOR Expression linear accelerator using phase space in the MCNPX code, Progress in Nuclear Energ70, 64 (2014).

[8] R. Kramer, J. W. Vieira, H. J. Khoury, F. R. A. Lima, D. Fuelle, All about Max: a Male Adult Voxel Phantom for Monte Carlo Calculation in Radiation Protection Dosimetry, Phys. Med. Biol bf 48, 123 (2003).

[9] I. G. Azubal, C. R. Harrell, E. O. Smith, A. L. Smith, P. Krischlunas, High resolution, MRI-based, segmented, computerized head phantom, In The Zubal Phantom Data, Voxel-Based Anthropomorphic Phantoms, (2001).

[10] ICRP 89, Reference Anatomical and Physiological Values in Radio protection, International Commission on Radiological Protection, Pergamon Press, Oxford, 2003.

[11] R. Kramer, H. J. Khoury, V. J. M. Lima, MAX06 and FAX06: update of two adult human phantoms for radiation protection dosimetry, Phys. Med. Biol. 51, 3331 (2006). 
[12] ICRP 103, The 2007 Recommendations of the International Commission on Radiological Protection, International Commission on Radiological Protection, Pergamon Press, Oxford, 2007.

[13] P. Cadman, N. P. S. Sidhu, Feasibility of penumbra compensating filters for conformal prostate radiotherapy, Phys. Med. Bio. 45, 295 (2000).

[14] J. P. Reis Junior, Validação do cálculo de dose e unidade monitor usando a técnica do espaço de fase no Monte Carlo, XV Congresso da Sociedade Brasileira de radioterapia, Fortaleza, 2013. 